\title{
THE WEIGHTS OF KIDNEYS IN LATE INTRA-UTERINE LIFE AND CHILDHOOD
}

\author{
BY \\ JOHN L. EMERY AND AVINASH MITHAL \\ From the Department of Pathology, the Children's Hospital, Sheffield
}

(RECEIVED FOR PUBLICATION JULY 28, 1960)

\begin{abstract}
Statistical tables including 50 and 95 percentile ranges are presented of the combined kidney weights compared with body weight, crown-rump length, and gestation age of foetuses and newborn children, and also a table of kidney weights related to post-natal age. The importance of relating kidney weight to more than one general body criterion in the perinatal period is illustrated.
\end{abstract}

It is difficult enough to estimate normality in living newborns and infants, but when we come to estimate the normal weights of organs from infants that have died in the perinatal period, largely from diseases not known to us, the difficulties are much greater. The early work in this field and the problems involved were well reviewed by Cruickshank and Miller (1924a and b) and Browne (1924).

The weights of different organs for the first few months of intra-uterine life were worked out by Clatworthy and Anderson in 1944. The present standard weights for older foetuses were established by Potter and Adair (1949) and Potter (1952). They grouped cases in such a way that crown-rump length and gestation age and body weight were brought together in the same groups. Their method, which follows that used by many of the earlier workers in this field (Giese, 1898; Ylppö, 1919 ; Feldman, 1920 ; Cruickshank and Miller, 1924b ; and Browne, 1924), is excellent for elucidating the general principles of differential growth of organs, but does not supply the particular information sometimes required by the pathologist.

For later childhood, the best established normal weights are those of Coppoletta and Wolbach (1933). Since then the general weight and height of children has increased both in Europe and America (Provis and Ellis, 1955; Stein, 1955; Thomson, 1956 ; Lancet, 1956). Keddie (1956) suggests that during the past 40 years there has been an increase of approximately $7 \frac{1}{2} \mathrm{lb}$. in weight and $3 \mathrm{in}$. in height in 9-year-old children.

The present paper is part of a general study being carried out in Sheffield to establish the normal weights of different organs of infants and children at the present time in England.

\section{Material and Methods}

The kidneys used in this study came from the records of 2,500 necropsies carried out by the Department of Pathology at the Children's Hospital, Sheffield, from 1950 to 1960 . Throughout this time the necropsies were carried out with the definite view of assessing the normal weights of organs. Kidneys were fixed uncut, while still attached to their blood vessels. After fixation the kidneys were separated from the ureters and blood vessels, and as much of the perirenal fat as possible was removed, but the capsule was not stripped. The kidneys were weighed together on a regularly tested Avery scale correct to the nearest half-gram.

All material was surveyed, and all cases showing abnormality of the renal tract, multiple congenital deformities, gross post-mortem changes, or histological abnormality of either kidney were eliminated. This left approximately 1,300 cases.

The combined weights of the kidneys were charted separately against crown-rump length, body weight (in grams), gestation age (estimated from the first day of the mother's last menstrual period) and post-natal age irrespective of gestation age at birth. These measurements were not complete in all cases, thus the number of cases used in the different correlations was not identical. Means, standard deviations, standard error of means based on the formula $\mathrm{SE}=\sqrt{ }^{\frac{\sigma}{\mathrm{n}}}$, and 50 and 95 percentile ranges were calculated.

\section{Results and Discussion}

A great variation in the range of weights of kidney was found for all criteria, but no greater than that found in the more recent studies on adult kidneys (Wald, 1937). The findings are given 
TABLE I

KIDNEY WEIGHT RELATED TO CROWN-RUMP LENGTH

\begin{tabular}{|c|c|c|c|c|c|}
\hline $\begin{array}{c}\text { Crown- } \\
\text { rump } \\
\text { Length } \\
\text { (cm.) }\end{array}$ & $\underset{\text { (g.) }}{\text { Mean }}$ & S.E. & $\begin{array}{c}50 \% \\
\text { Range }\end{array}$ & $\begin{array}{c}95 \% \\
\text { Range }\end{array}$ & $\begin{array}{l}\text { Potter } \\
\text { and } \\
\text { Adair } \\
\text { (1949) }\end{array}$ \\
\hline $\begin{array}{l}22 \\
23 \\
24 \\
25 \\
26 \\
27 \\
28 \\
29 \\
30 \\
31 \\
32 \\
33 \\
34 \\
35 \\
36 \\
37 \\
38 \\
39 \\
40 \\
41 \\
42 \\
43 \\
44 \\
45 \\
46 \\
47 \\
48 \\
49 \\
50\end{array}$ & $\begin{array}{r}7 \cdot 0 \\
8 \cdot 5 \\
9 \cdot 5 \\
11 \cdot 0 \\
12 \cdot 5 \\
14 \cdot 0 \\
15 \cdot 5 \\
17 \cdot 0 \\
18 \cdot 5 \\
20 \cdot 0 \\
21 \cdot 5 \\
23 \cdot 0 \\
25 \cdot 0 \\
26 \cdot 5 \\
28 \cdot 5 \\
30 \cdot 5 \\
33 \cdot 0 \\
36 \cdot 0 \\
39 \cdot 0 \\
41 \cdot 5 \\
45 \cdot 5 \\
50 \cdot 0 \\
53 \cdot 5 \\
57 \cdot 0 \\
61 \cdot 5 \\
65 \cdot 0 \\
69 \cdot 0 \\
73 \cdot 0 \\
77 \cdot 0\end{array}$ & $\begin{array}{l}0.65 \\
0.65 \\
0.55 \\
0.55 \\
0.55 \\
0 \cdot 3 \\
0 \cdot 3 \\
0.3 \\
1.4 \\
1.12 \\
0.85 \\
0.88 \\
0.92 \\
0.94 \\
0.94 \\
1.37 \\
1.37 \\
1.48 \\
1.48 \\
1.48 \\
1.48 \\
1.48 \\
3.75 \\
3.75 \\
3.75 \\
3.75 \\
3.75 \\
3.75 \\
3.75\end{array}$ & $\begin{array}{r}4 \cdot 5-8 \cdot 0 \\
5 \cdot 5-9 \cdot 5 \\
7 \cdot 0-11 \cdot 5 \\
8 \cdot 0-13 \cdot 0 \\
9 \cdot 5-15 \cdot 0 \\
11 \cdot 0-17 \cdot 0 \\
12 \cdot 5-19 \cdot 0 \\
14 \cdot 0-20 \cdot 5 \\
16 \cdot 0-22 \cdot 5 \\
17 \cdot 0-24 \cdot 5 \\
18 \cdot 5-26 \cdot 0 \\
20 \cdot 0-28 \cdot 5 \\
21 \cdot 5-30 \cdot 5 \\
23 \cdot 0-32 \cdot 0 \\
24 \cdot 0-34 \cdot 0 \\
26 \cdot 5-36 \cdot 0 \\
27 \cdot 0-38 \cdot 0 \\
28 \cdot 0-40 \cdot 5 \\
30.0-43 \cdot 0 \\
32 \cdot 5-46 \cdot 0 \\
36 \cdot 0-50 \cdot 0 \\
40 \cdot 0-53 \cdot 5 \\
43 \cdot 0-58 \cdot 0 \\
48 \cdot 0-61 \cdot 5 \\
51 \cdot 0-65 \cdot 5 \\
54 \cdot 0-69 \cdot 5 \\
57 \cdot 0-73 \cdot 5 \\
60 \cdot 5-77 \cdot 5 \\
64 \cdot 0-82 \cdot 0\end{array}$ & $\begin{array}{c}2 \cdot 0-11 \cdot 0 \\
3 \cdot 0-12 \cdot 0 \\
4 \cdot 0-15 \cdot 0 \\
5 \cdot 0-16 \cdot 0 \\
5 \cdot 5-17 \cdot 5 \\
6 \cdot 5-19 \cdot 5 \\
7 \cdot 0-21 \cdot 5 \\
7 \cdot 5-23 \cdot 5 \\
8 \cdot 0-26 \cdot 0 \\
8 \cdot 5-28 \cdot 0 \\
9 \cdot 0-31 \cdot 0 \\
10 \cdot 0-32 \cdot 5 \\
10 \cdot 0-35 \cdot 0 \\
11 \cdot 0-38 \cdot 0 \\
12 \cdot 0-41 \cdot 0 \\
13 \cdot 0-45 \cdot 0 \\
14 \cdot 0-48 \cdot 0 \\
14 \cdot 5-52 \cdot 5 \\
15 \cdot 5-56 \cdot 5 \\
16 \cdot 5-61 \cdot 0 \\
18 \cdot 0-66 \cdot 0 \\
19 \cdot 0-71 \cdot 0 \\
21 \cdot 0-75 \cdot 5 \\
22 \cdot 5-81 \cdot 5 \\
24 \cdot 0-88 \cdot 0 \\
26 \cdot 0-93 \cdot 0 \\
28 \cdot 0-99 \cdot 0 \\
30 \cdot 0-105 \cdot 0 \\
32 \cdot 5-113 \cdot 0\end{array}$ & $\begin{array}{l}18 \cdot 3 \\
21 \cdot 1 \\
23 \cdot 6 \\
26 \cdot 6 \\
29 \cdot 3 \\
32 \cdot 2\end{array}$ \\
\hline
\end{tabular}

TABLE II

KIDNEY WEIGHT RELATED TO TOTAL BODY WEIGHT

\begin{tabular}{|c|c|c|c|c|c|}
\hline $\begin{array}{c}\text { Body } \\
\text { Weight } \\
\text { (g.) }\end{array}$ & Mean & S.E. & $\begin{array}{c}50 \% \\
\text { Range }\end{array}$ & $\begin{array}{c}95 \% \\
\text { Range }\end{array}$ & $\begin{array}{l}\text { Potter } \\
\text { and } \\
\text { Adair } \\
\text { (1949) }\end{array}$ \\
\hline $\begin{array}{l}1,400 \\
1,500 \\
1,600 \\
1,700 \\
1,800 \\
1,900 \\
2,000 \\
2,100 \\
2,200 \\
2,300 \\
2,400 \\
2,500 \\
2,600 \\
2,700 \\
2,800 \\
2,900 \\
3,000 \\
3,100 \\
3,200 \\
3,300 \\
3,400 \\
3,500 \\
3,600 \\
3,700 \\
3,800 \\
3,900 \\
4,000\end{array}$ & $\begin{array}{l}15 \cdot 5 \\
17 \cdot 0 \\
18 \cdot 0 \\
19 \cdot 0 \\
19 \cdot 8 \\
20 \cdot 5 \\
21 \cdot 0 \\
22.0 \\
23 \cdot 0 \\
23 \cdot 5 \\
24 \cdot 0 \\
25 \cdot 0 \\
25 \cdot 5 \\
26 \cdot 0 \\
27 \cdot 0 \\
27 \cdot 5 \\
28 \cdot 5 \\
29 \cdot 0 \\
29 \cdot 5 \\
30 \cdot 0 \\
31 \cdot 0 \\
31 \cdot 5 \\
32 \cdot 5 \\
33 \cdot 0 \\
33 \cdot 5 \\
34 \cdot 5 \\
35 \cdot 0\end{array}$ & $\begin{array}{l}1.03 \\
1.03 \\
1.03 \\
0.83 \\
0.83 \\
0.83 \\
0.83 \\
0.83 \\
1.24 \\
1.24 \\
1.24 \\
0.81 \\
0.81 \\
0.81 \\
0.98 \\
0.98 \\
0.98 \\
0.98 \\
1.46 \\
1.46 \\
1.46 \\
1.27 \\
1.27 \\
1.27 \\
1.27 \\
1.27 \\
2.00\end{array}$ & $\begin{array}{l}14 \cdot 0-20 \cdot 5 \\
14 \cdot 5-21 \cdot 0 \\
15 \cdot 5-21 \cdot 5 \\
16 \cdot 0-22 \cdot 0 \\
16 \cdot 5-23 \cdot 0 \\
17 \cdot 5-23 \cdot 5 \\
18 \cdot 5-24 \cdot 5 \\
19 \cdot 0-25 \cdot 0 \\
19 \cdot 5-25 \cdot 5 \\
20 \cdot 5-26 \cdot 5 \\
21 \cdot 0-27 \cdot 0 \\
22 \cdot 0-28 \cdot 0 \\
22 \cdot 5-29 \cdot 0 \\
22 \cdot 5-30 \cdot 0 \\
23 \cdot 0-31 \cdot 0 \\
23 \cdot 0-32 \cdot 0 \\
23 \cdot 5-33 \cdot 0 \\
23 \cdot 5-34 \cdot 0 \\
24 \cdot 0-34 \cdot 5 \\
24 \cdot 5-35 \cdot 5 \\
25 \cdot 0-36 \cdot 0 \\
26 \cdot 0-36 \cdot 5 \\
26 \cdot 5-37 \cdot 0 \\
27 \cdot 5-38 \cdot 0 \\
28 \cdot 0-38 \cdot 5 \\
29 \cdot 0-39 \cdot 0 \\
30 \cdot 0-40 \cdot 5\end{array}$ & $\begin{array}{r}9 \cdot 5-25 \cdot 5 \\
10 \cdot 0-26 \cdot 0 \\
10 \cdot 5-27 \cdot 0 \\
11 \cdot 0-27 \cdot 5 \\
11 \cdot 5-28 \cdot 0 \\
12 \cdot 0-29 \cdot 0 \\
13 \cdot 0-30 \cdot 0 \\
13 \cdot 5-31 \cdot 0 \\
14 \cdot 0-32 \cdot 0 \\
14 \cdot 0-33 \cdot 0 \\
14.5-34 \cdot 5 \\
15 \cdot 0-35 \cdot 0 \\
15 \cdot 0-35 \cdot 5 \\
15 \cdot 0-37 \cdot 0 \\
15 \cdot 0-38 \cdot 0 \\
15 \cdot 5-39 \cdot 0 \\
15 \cdot 5-40 \cdot 0 \\
155-41 \cdot 5 \\
16 \cdot 0-42 \cdot 5 \\
16 \cdot 5-43 \cdot 5 \\
17 \cdot 0-45 \cdot 0 \\
17 \cdot 5-46 \cdot 0 \\
18 \cdot 0-47 \cdot 0 \\
18 \cdot 0-48 \cdot 5 \\
18.5-49 \cdot 0 \\
19 \cdot 0-50 \cdot 5 \\
20 \cdot 0-52 \cdot 0\end{array}$ & $21 \cdot 1$ \\
\hline
\end{tabular}

in Tables I to IV. Comparative figures, where available, are included. Kidney weights graphed against post-natal age are also presented in Fig. 1.

It is thought essential that the weights be presented separately, against body weight, gesta- tion age, and length, and not combined, as Potter and Adair found convenient, due to the differences found in the variation from "normal" under different criteria. This is illustrated in Fig. 2, where the scatter diagrams of the kidney weights against crown-rump length and body weight are shown. In the chart using the total weight of the child, three cases, A, B, and C, are well outside the 95 percentile ranges. The same cases in the crownrump length chart are not only well within the 95 percentiles, but close together. Thus, against body weight, these body weights are grossly abnormal, whereas against crown-rump length they are quite normal.

TABLE III

KIDNEY WEIGHT RELATED TO GESTATION AGE

\begin{tabular}{|c|c|c|c|c|c|}
\hline $\begin{array}{c}\text { Gesta- } \\
\text { tion } \\
\text { Age } \\
\text { (wk.) }\end{array}$ & Mean & S.E. & $\begin{array}{l}50 \% \\
\text { Range }\end{array}$ & $\begin{array}{l}95 \% \\
\text { Range }\end{array}$ & $\begin{array}{l}\text { Potter } \\
(1952)\end{array}$ \\
\hline $\begin{array}{l}28 \\
29 \\
30 \\
31 \\
32 \\
33 \\
34 \\
35 \\
36 \\
37 \\
38 \\
39 \\
40 \\
41 \\
42 \\
43 \\
44 \\
45\end{array}$ & $\begin{array}{l}11 \cdot 0 \\
12.0 \\
13.0 \\
14.5 \\
16.0 \\
17 \cdot 0 \\
18 \cdot 0 \\
19.5 \\
20.5 \\
21 \cdot 5 \\
23 \cdot 0 \\
24 \cdot 0 \\
25 \cdot 5 \\
27.0 \\
28 \cdot 0 \\
29.5 \\
30.5 \\
31.5\end{array}$ & $\begin{array}{l}2 \cdot 49 \\
2 \cdot 29 \\
2 \cdot 20 \\
2 \cdot 16 \\
2.75 \\
3.33 \\
3.97 \\
4.61 \\
350 \\
3 \cdot 50 \\
3 \cdot 50 \\
2 \cdot 38 \\
2.87 \\
3.36 \\
4.41 \\
5.46 \\
6.12 \\
6.79\end{array}$ & $\begin{array}{r}9 \cdot 5-13 \cdot 5 \\
10 \cdot 0-14 \cdot 5 \\
11 \cdot 0-16.0 \\
12 \cdot 0-17 \cdot 0 \\
13 \cdot 0-18 \cdot 5 \\
14 \cdot 0-19 \cdot 5 \\
15 \cdot 0-20 \cdot 5 \\
16 \cdot 5-22 \cdot 0 \\
17 \cdot 0-23 \cdot 0 \\
18 \cdot 0-24 \cdot 5 \\
19 \cdot 0-26 \cdot 0 \\
20 \cdot 0-27 \cdot 5 \\
21 \cdot 0-29 \cdot 5 \\
21 \cdot 5-31 \cdot 0 \\
22 \cdot 5-32 \cdot 5 \\
23 \cdot 5-34 \cdot 5 \\
24 \cdot 5-36 \cdot 0 \\
26.0-38 \cdot 0\end{array}$ & $\begin{array}{r}4 \cdot 5-19 \cdot 5 \\
5 \cdot 0-21 \cdot 0 \\
5 \cdot 5-22 \cdot 5 \\
6 \cdot 0-24 \cdot 0 \\
6 \cdot 5-25 \cdot 5 \\
7 \cdot 0-26 \cdot 5 \\
7 \cdot 5-27 \cdot 5 \\
8 \cdot 5-30 \cdot 0 \\
90-31 \cdot 5 \\
9 \cdot 5-33 \cdot 5 \\
10 \cdot 0-35 \cdot 5 \\
11 \cdot 0-37 \cdot 5 \\
11 \cdot 5-39 \cdot 5 \\
12 \cdot 5-41 \cdot 0 \\
13 \cdot 0-43 \cdot 0 \\
14 \cdot 0-45 \cdot 0 \\
15.0-47 \cdot 0 \\
16.0-49 \cdot 0\end{array}$ & $\begin{array}{l}18 \cdot 3 \\
21 \cdot 1 \\
23 \cdot 6 \\
26 \cdot 6 \\
32 \cdot 2\end{array}$ \\
\hline
\end{tabular}

TABLE IV

KIDNEY WEIGHT RELATED TO POST-NATAL AGE

\begin{tabular}{|c|c|c|c|c|c|c|}
\hline Age & Mean & S.E. & $\begin{array}{c}50 \% \\
\text { Range }\end{array}$ & $\begin{array}{c}95 \% \\
\text { Range }\end{array}$ & $\begin{array}{c}\text { Coppol- } \\
\text { etta } \\
\text { and } \\
\text { Wolbach } \\
(1933)\end{array}$ & $\begin{array}{c}\text { Tkate- } \\
\text { schenko } \\
\text { (1900) }\end{array}$ \\
\hline 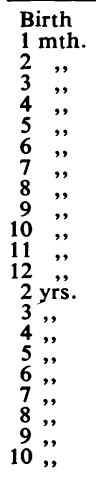 & $\begin{array}{r}26 \\
32 \\
36 \\
39 \\
44 \\
48 \\
51 \\
56 \\
60 \\
62 \\
67 \\
69 \\
78 \\
88 \\
100 \\
110 \\
121 \\
134 \\
144 \\
156 \\
166 \\
178\end{array}$ & $\begin{array}{l}0.76 \\
0.98 \\
1.13 \\
1.43 \\
1.73 \\
1.73 \\
1.73 \\
2.04 \\
2.04 \\
2.04 \\
2.04 \\
2.04 \\
2.04 \\
2.74 \\
5.2 \\
5.2 \\
5.2 \\
6.4 \\
6.4 \\
6.4 \\
6.4 \\
6.4\end{array}$ & $\begin{array}{r}22-34 \\
26-38 \\
29-42 \\
33-46 \\
36-50 \\
40-54 \\
43-58 \\
46-63 \\
50-68 \\
54-72 \\
58-78 \\
63-85 \\
68-92 \\
74-103 \\
81-114 \\
92-126 \\
100-136 \\
110-150 \\
120-160 \\
131-173 \\
142-185 \\
153-196\end{array}$ & $\begin{array}{r}13-44 \\
16-50 \\
18-54 \\
21-60 \\
24-65 \\
27-70 \\
30-76 \\
32-82 \\
35-88 \\
38-95 \\
40-100 \\
43-109 \\
48-118 \\
54-130 \\
62-142 \\
72-156 \\
81-169 \\
90-182 \\
101-194 \\
111-207 \\
120-220 \\
130-230\end{array}$ & $\begin{array}{l}27 \\
30 \\
37 \\
39 \\
43 \\
50 \\
51 \\
60 \\
61 \\
61 \\
63 \\
67 \\
71 \\
93 \\
97 \\
114 \\
129 \\
135 \\
139 \\
149 \\
165 \\
187\end{array}$ & $\begin{array}{c}22-24 \\
22 \cdot 8-24 \cdot 8 \\
32-36 \\
45 \cdot 2-47 \cdot 2 \\
58-60 \\
58-60 \\
72-74 \\
95-100 \\
110-112\end{array}$ \\
\hline
\end{tabular}




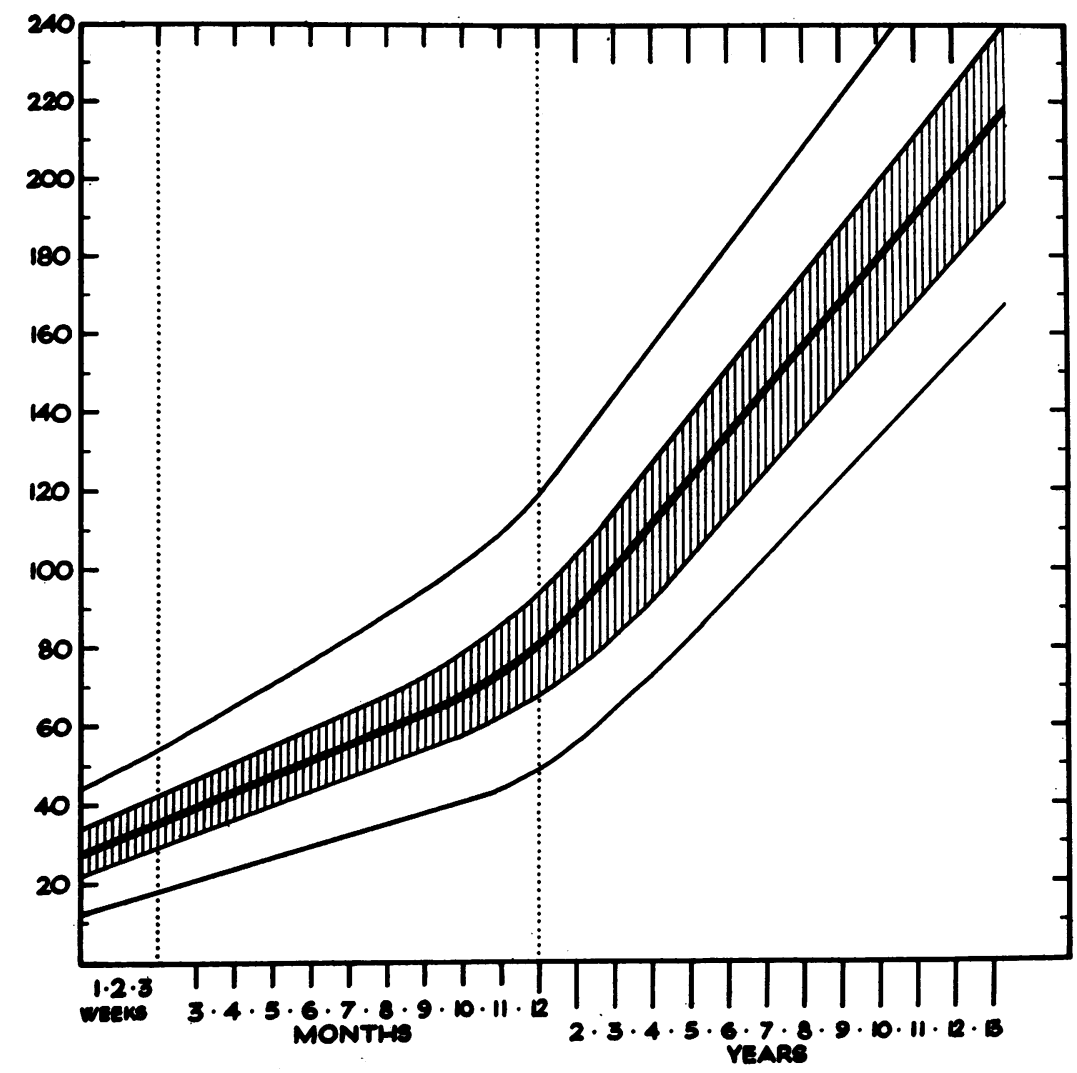

FIG. 1.-The mean combined kidney weights, the 50 percentile range (shaded), and the 95 percentiles (unshaded), the kidney weight being related to post-natal age, from 804 pairs of kidneys.

The method of estimation of the normal range used is that of percentiles rather than standard deviation. This problem was discussed well by Boyd (1935), who concludes that percentile ranges are the most useful as was originally stressed by Sir Francis Galton in 1885.

Our findings for post-natal kidney weight show slightly lower values than those of Coppoletta and Wolbach (1933). This is surprising because our method did not permit the kidneys to be squeezed and emptied of blood before weighing. Also, the general body weight and height of these children are probably higher than those of children used in the Boston series of 25 years ago.

The foetal kidney weights given by Cruickshank and Miller (1924b) and Browne (1924), from this country, compare very closely with our figures, suggesting that foetal kidney weights have not altered over the last 25 years. In general our weights show a higher range than those of Potter and Adair (1949) and Potter (1952), but their presentation is not strictly comparable to the present study as they produced common body weight, age, and body length grouping. We have shown that quite different results can be obtained when the kidney weight is related to the total body weight than when related to crown-rump length, and the same applies to gestation age.

When attempts are made to elucidate intrauterine disease, estimates should be made against the different general body measurements separately.

Much of the material used in this study came from necropsies carried out through the courtesy of Dr. N A. J. Warrack. Dr. Avinash Mithal is working under a grant from the Medical Research Council.

\section{REFERENCES}

Boyd, E. (1935). Anat. Rec., 62, 1.

Browne, F. J. (1924). Spec. Rep. Ser. med. Res. Coun. (Lond.), No.

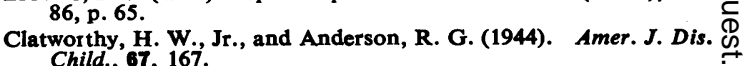
Child., 67, 167.

Coppoletta, J. M., and Wolbach, S. B. (1933). Amer. J. Path., 9, 55. प Cruickshank, J. N., and Miller, M. J. (1924a). Spec. Rep. Ser. med. $\bar{O}$ Res. Coun. (Lond.), No. 86, p. 5. (1924b). Ibid., p. 33. 

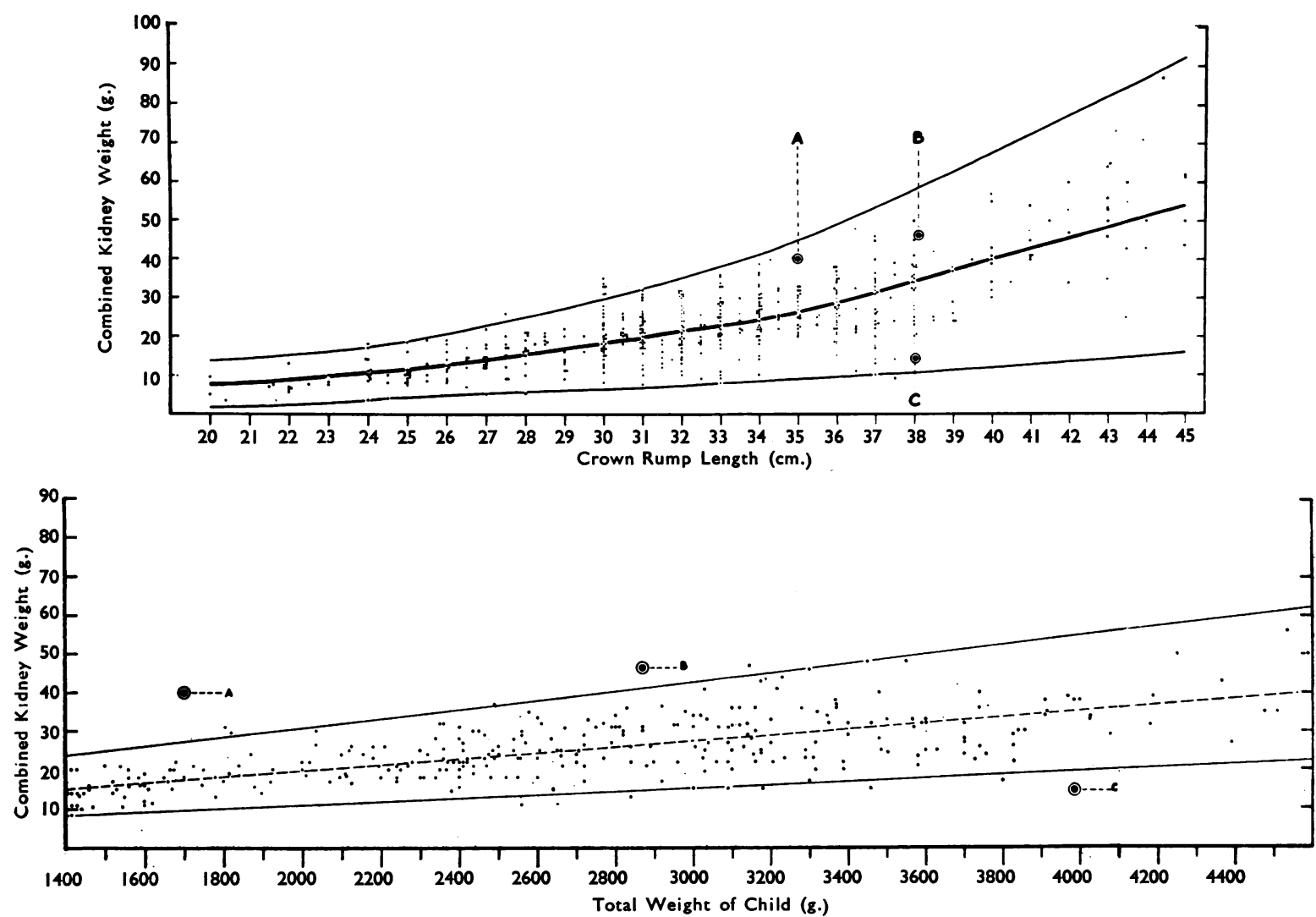

Fig. 2.-Scatter diagrams of the combined kidney weights with the means and 95 percentile lines against crown-rump length and total weight of the child in grams. The points marked A, B, and C represent the same kidneys as they fall in the different graphs.

Feldman, W. M. (1920). The Principles of Ante-natal and Post-natal Child Physiology, p. 503. Longmans, Green, London.

Galton, F. (1885). J. roy. Anthrop. Inst., 14, 275.

Giese, E. (1898). Inaug. Dissert., St. Petersburg. Quoted by Feldman (1920), p. 225.

Keddie, J. A. G. (1956). Brit. J. prev. soc. Med., 10, 1.

Lancet (1956), 2, 183 (Annotation).

Potter, E. L. (1952). Pathology of the Fetus and the Newborn. Year Book Publishers, Chicago.
Potter, E. L., and Adair, F. L. (1949). Fetal and Neonatal Death. Second ed. Univ. Chicago Press, Chicago.

Provis, H. S., and Ellis, R. W. B. (1955). Arch. Dis. Childh., 30, 328.

Stein, L. (1955). Ibid., 30, 432.

Thomson, J. (1956). Ibid., 31, 382.

Tkateschenko (1900). Inaug. Dissert., St. Petersburg. Quoted by Feldman (1920), p. 502.

Wald, H. (1937). Arch. Path. (Chicago), 23, 493.

Ylppö, A. (1919). Z. Kinderheilk., 20, 212. 